\title{
Quantum entanglement of locally excited states in Maxwell theory
}

\author{
Masahiro Nozaki ${ }^{a}$ and Naoki Watamura ${ }^{b}$ \\ ${ }^{a}$ Kadanoff Center for Theoretical Physics, University of Chicago, \\ Chicago, Illinois 60637, U.S.A. \\ ${ }^{b}$ Department of Physics, Nagoya University, \\ Nagoya 464-8602, Japan \\ E-mail: masahiron@uchicago.edu, watamura@eken.phys.nagoya-u.ac.jp
}

ABstract: In 4 dimensional Maxwell gauge theory, we study the changes of (Rényi) entanglement entropy which are defined by subtracting the entropy for the ground state from the one for the locally excited states, generated by acting with gauge invariant local operators on the state. The changes for the operators which we consider in this paper reflect the electric-magnetic duality. The late-time value of changes can be interpreted in terms of electromagnetic quasi-particles. When the operator constructed of both electric and magnetic fields acts on the ground state, it shows that the operator acts on the late-time structure of quantum entanglement differently from free scalar fields.

KEYwords: Gauge Symmetry, Duality in Gauge Field Theories, Conformal Field Theory ARXIV EPrint: 1606.07076 


\section{Contents}

1 Introduction and summary 1

2 How to compute excesses of (Rényi) entanglement entropy 3

2.1 The replica trick 4

3 Excesses of (Rényi) entanglement entropy $\quad 7$

$3.1 \mathcal{O}=E_{i}$ or $B_{i} \quad 8$

3.2 Composite operators constructed of only electric or magnetic fields 9

3.2.1 $\mathcal{O}=E_{i} E_{j}$ or $B_{i} B_{j} \quad 9$

$\begin{array}{ll}3.2 .2 \mathcal{O}=\mathbf{E}^{2} \text { or } \mathbf{B}^{2} & 11\end{array}$

$\begin{array}{lll}3.3 & \text { Composite operators constructed of both electric and magnetic fields } & 12\end{array}$

$\begin{array}{lll}3.3 .1 & E_{1}^{2}+B_{1}^{2} & 12\end{array}$

$\begin{array}{lll}3.3 .2 & E_{i} B_{j} & 13\end{array}$

$\begin{array}{lll}3.3 .3 & \mathbf{B} \cdot \mathbf{E} \text { and } F_{\mu \nu} F^{\mu \nu} \text { and } B_{2} E_{3}-B_{3} E_{2} & 13\end{array}$

$\begin{array}{lll}4 \text { A late-time algebra } & 14\end{array}$

5 Conclusion and future problems $\quad 16$

$\begin{array}{ll}\text { A Green's functions } & 17\end{array}$

$\begin{array}{ll}\text { B Other bases } & 19\end{array}$

\section{Introduction and summary}

Quantum entanglement significantly distinguishes quantum states from classical states. It can characterize conformal field theories [1-4] and topological phases [5-7]. In Gauge/Gravity correspondence [8-10], the structure of quantum entanglement in quantum field theories (QFTs) living on the boundary is expected to be related to the gravity in the bulk [11-14]. There has been a lot of work done to reveal how the structure of quantum entanglement on the boundary corresponds to the geometry in the bulk [15-25]. Therefore it is important to uncover the fundamental features which quantum entanglement possesses. (Rényi) entanglement entropy is one of the useful quantities to investigate them.

However the definition of (Rényi) entanglement entropy in gauge theories has subtleties [26-41]. In gauge theories, physical states have to be gauge invariant. They obey constraints which guarantee their gauge invariance. They make it difficult to divide the Hilbert space into subsystems $A$ and $B$ because the physical degrees of freedom in $A$ depends on the freedom in $B$ due to the constraints. Their boundary is $\partial A$. Then the definition of (Rényi) entanglement entropy needs a precise method of dividing Hilbert space 
and defining the reduced density matrix $\rho_{A}$ which is given by tracing out the degrees of freedom in $B$,

$$
\rho_{A}=\operatorname{tr}_{B} \rho
$$

On the other hand, the entropy in QFTs depends on a UV cutoff (ultraviolet cutoff) $\delta$ because by definition it has the UV divergence. It is given by a series expansion in conformal field theories. The physical degrees of freedom around $\partial A$ have the significant effect on the terms which depend on $\delta$. The method of dividing the Hilbert space is expect to affect the degrees of freedom around $\partial A$ in the direct fashion. In the present paper, we study the changes of (Rényi) entanglement entropy $\Delta S_{A}^{(n)}$ which is defined by subtracting the entropy for the ground state from the one for the locally excited state, which is defined by acting with a local operator on the ground state. Here we assume that the operator is located far from $\partial A$. We will explain it more in the next section. As in [42-48], their changes do not possess the UV divergence. More precisely, they measure how the local operator changes the structure of quantum entanglement. Therefore, they are expected to avoid the subtleties which (Rényi) entanglement entropy has.

In this paper we study $\Delta S_{A}^{(n)}$ in $4 d$ Maxwell gauge theory, which is a free CFT [49]. The previous works [44-46] show the time evolution of $\Delta S_{A}^{(n)}$ can be interpreted in terms of relativistic propagation of entangled quasi-particles which are created by local operators. In the free theories, the late-time value of $\Delta S_{A}^{(n)}$ is given by a constant, which depends on the operators. It comes from the quantum entanglement between quasi-particles. As in [46], the late-time entanglement structure depends on the kind of quasi-particles. The authors in [47] show that in the specific $2 d$ CFTs, it is related to the quantum dimension of the operator which acts on the ground state. The authors in $[48,50]$ have shown that in holographic theories the late time value of $\Delta S_{A}^{(n)}$ logarithmically increases similarly to the behavior of entanglement entropy for the local quenches [51, 52]. $\Delta S_{A}^{(n)}$ in the finite temperature system was investigated by the authors in [53]. Many works have been done to study the fundamental properties of $\Delta S_{A}^{(n)}$ [54-59]. The time evolution and late-time value of $\Delta S_{A}^{(n)}$ depend on theories and the quasi-particles created by the local operator. Then we study how the structure of quantum entanglement is changed by gauge invariant local operators such as electric and magnetic fields. In particular, we study how the late-time structure of quantum entanglement depends on them. More precisely, we study the effect of quasi-particles on the structure. We also study whether $\Delta S_{A}^{(n)}$ for a gauge invariant locally excited state reflects electric-magnetic duality.

Summary. Here, we briefly summarize our results in this work. We study how the various local gauge invariant operators change the structure of quantum entanglement by measuring the time evolution of $\Delta S_{A}^{(n)}$ for various gauge invariant local operators. We also study whether $\Delta S_{A}^{(n)}$ is invariant under the electric-magnetic duality transformation.

Electric-magnetic duality. As it will be explained later, $\Delta S_{A}^{(n)}$ for locally excited states is invariant under the transformation $E_{i} \rightarrow-B_{i}$ and $B_{i} \rightarrow E_{i}$ where $E$ and $B$ are electric and magnetic fields, respectively. 
$\boldsymbol{E}_{\boldsymbol{i}}$ or $\boldsymbol{B}_{\boldsymbol{i}} . \quad$ If only $E_{i}$ or $B_{i}$ acts on the ground state, the time evolution of $\Delta S_{A}^{(n)}$ depends on the one which acts on the ground state. Because $\Delta S_{A}^{(n)}$ reflects the electric-magnetic duality, the entropy for $B_{i}$ is equal to that for $E_{i}$, which is the electric field along the same direction as that of magnetic field. $\Delta S_{A}^{(n)}$ for the electric and magnetic fields along the direction vertical to the entangling surface increases slower than those for fields along the directions parallel to the surface. However there are no differences between the effects of electromagnetic field and that of scalar one on the entanglement structure at the late time. ${ }^{1}$

Composite operators. If the composite operator such as $\mathbf{B}^{2}$ acts on the ground state, they lead to the late-time structure of quantum entanglement in the same manner as a specific scalar operator. Then the late-time value of $\Delta S_{A}^{(n)}$ for that can be interpreted in terms of quasi-particles created by the scalar operator. However $\Delta S_{A}^{(n)}$ for some specific operators (e.g, $\left.E_{2} B_{3}\right)$ constructed of both electric and magnetic fields can not be interpreted in terms of the scalar quasi-particles but of electromagnetic ones, which is explained in section 4. Here $B_{3}\left(E_{3}\right)$ and $B_{2}\left(E_{2}\right)$ are the magnetic (electric) fields along the direction perpendicular to $\partial A$ as we will explain it later.

Late-time algebra. We interpret the late-time values of $\Delta S_{A}^{(n)}$ in terms of electromagnetic quasi-particles created by an electromagnetic field, and derive a late-time algebra which they obey. There are commutation relations between the particles of the same kinds of fields. As we will show later, there are also additional relations between $E_{2}\left(E_{3}\right)$ and $B_{3}\left(B_{2}\right)$, which are parallel to the entangling surface. They make the effect of electromagnetic fields different from that of scalar fields on the late-time structure of quantum entanglement.

Organization. This paper is organized as follows. In section 2, we will explain locally excited states and how to compute $\Delta S_{A}^{(n)}$ in the replica trick. We study the time evolution and late-time value of $\Delta S_{A}^{(n)}$ for various gauge invariant local operators in section 3. We interpret the late-time value of $\Delta S_{A}^{(n)}$ in terms of entangled quasi-particles in section 4 . We study how they have the effect on the late-time structure of quantum entanglement. We finish with the conclusion, future problems and the detail of propagators is included in appendices.

\section{How to compute excesses of (Rényi) entanglement entropy}

By measuring the excess of (Rényi) entanglement entropies $\Delta S_{A}^{(n)}$, we study how local gauge-invariant operators changes the structure of quantum entanglement in the $4 d$ Maxwell gauge theory:

$$
S=-\frac{1}{4} \int d^{4} x F_{\mu \nu} F_{\rho \sigma} g^{\mu \rho} g^{\nu \sigma}
$$

where $F_{\mu \nu}=\partial_{\mu} A_{\nu}-\partial_{\nu} A_{\mu}$ and $g^{\mu \nu}=\operatorname{diag}(-1,1,1,1)$.

\footnotetext{
${ }^{1}$ When only a component of the electric or magnetic one acts on the ground state, we do not consider the linear combination of them in this paper.
} 


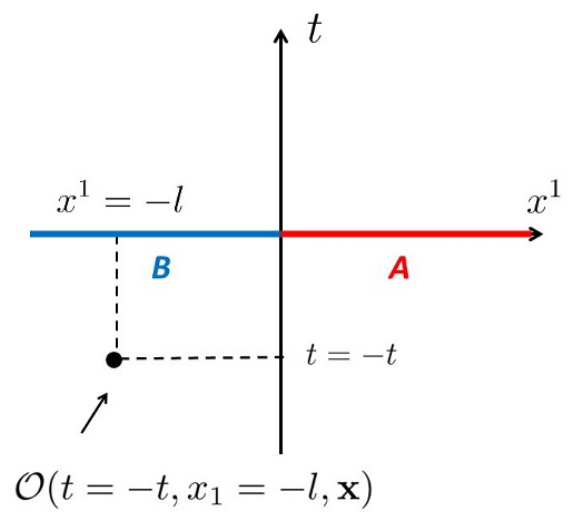

Figure 1. The location of local gauge invariant operator in Minkowski spacetime.

In this section, we explain the definition of locally excited state and how to compute $\Delta S_{A}^{(n)}$ in the replica method.

The definition of locally excited states. The locally excited state is defined by acting with a gauge invariant local operator $\mathcal{O}$ such as $F^{\mu \nu}$ on the ground state:

$$
|\Psi\rangle=\mathcal{N O}(-t,-l, \mathbf{x})|0\rangle
$$

where $\mathcal{N}$ is a normalization constant and $|0\rangle$ is a gauge invariant state. As in figure $1, \mathcal{O}$ is located at $t=-t, x^{1}=-l$ and $\mathbf{x}=\left(x^{2}, x^{3}\right)$.

Subsystem. As in the previous works [44-47], the subsystem $A$ is defined by $(t=0$, $\left.x^{1} \geq 0\right)$ as in figure 1 . In free theories $\Delta S_{A}^{(n)}$ approaches to a constant, which comes from quantum entanglement between entangled quasi-particles. In this paper we would like to study how the constant depends on gauge invariant operators. Therefore the region in figure 1 is chosen as $A$.

Excesses of (Rényi) entanglement entropy. Here we explain more about the definition of $\Delta S_{A}^{(n)}$. (Rényi) entanglement entropy for the ground state is a static quantity, which does not depend on time. Then we define the excesses of (Rényi) entanglement entropy $\Delta S_{A}^{(n)}$ by subtracting $S_{A}^{(n)}$ for the ground state from those for locally excited states,

$$
\Delta S_{A}^{(n)}=S_{A}^{(n), E X}-S_{A}^{(n), G}
$$

where $S_{A}^{(n), E X}$ and $S_{A}^{(n), G}$, are (Rényi) entanglement entropies for the excited states in (2.2) and the ground state $|0\rangle$, respectively. In the sense that $\Delta S_{A}^{(n)}$ does not depend on $\delta$, it is a "renormalized" (Rényi) entanglement entropy.

\subsection{The replica trick}

We would like to study the time evolution of $\Delta S_{A}^{(n)}$ in $4 d$ Minkowski spacetime. However in this paper we do not directly study the changes of entanglement structure in the spacetime. Instead, we compute $\Delta S_{A}^{(n)}$ in Euclidean space by the replica trick. After that we perform 
the analytic continuation, which we will explain later. Then we compute the real time evolution of $\Delta S_{A}^{(n)}$.

As in [44-47], a reduced density matrix in Euclidean space is given by

$$
\rho=\tilde{\mathcal{N}}^{2} \mathcal{O}\left(\tau_{e},-l, \mathbf{x}\right)|0\rangle\langle 0| \mathcal{O}^{\dagger}\left(\tau_{l},-l, \mathbf{x}\right),
$$

where $\tau$ is Euclidean time. By introducing a polar coordinate, $\left(\tau_{l, e},-l\right)$ is mapped to $\left(r_{1,2}, \theta_{1,2}\right)$ as in figure 2 .

The reduced density matrix of $\rho$ introduced above, which is our interest now, can be written in the form of path integral

$$
\left[\rho_{A}\right]_{\Phi_{1}, \Phi_{2}}=\left(Z_{1}^{E X}\right)^{-1} \int D \Phi \mathcal{O}^{\dagger}\left(x_{1}\right) \mathcal{O}\left(x_{2}\right) e^{-S[\Phi]} \delta\left(\Phi\left(-\Delta, x^{i}\right)-\Phi_{1}\left(x^{i}\right)\right) \cdot \delta\left(\Phi\left(\Delta, x^{i}\right)-\Phi_{2}\left(x^{i}\right)\right)
$$

where the indices $i, \mu$ is $i=1,2,3, \mu=0,1,2,3$, and $x^{i}$ runs over the region $\mathrm{A}$, which is in this case $x^{1}>0$ and $x^{2}, x^{3} \in \mathbf{R}$. The $\Phi_{1}=\Phi_{1}\left(x^{i}\right)$ and $\Phi_{2}=\Phi_{2}\left(x^{i}\right)$ introduced in the path integral inside the $\delta$-function with a positive value $\Delta \ll 1$ (which is taken to the zero limit in the end), represent the boundary condition at region A. $Z_{1}^{E X}$ is defined as

$$
Z_{1}^{E X}=\int D \Phi \mathcal{O}^{\dagger}\left(x_{1}\right) \mathcal{O}\left(x_{2}\right) e^{-S[\Phi]} .
$$

The $n$-th power of the reduced density matrix is written as

$$
\begin{aligned}
{\left[\rho_{A}^{n}\right]_{\Phi_{1}, \Phi_{2}}=} & \left(Z_{1}^{E X}\right)^{-n} \int D \Phi\left(\prod_{l=1}^{n} \mathcal{O}^{\dagger}\left(x_{1}^{l}\right) \mathcal{O}\left(x_{2}^{l}\right)\right) e^{-S_{n}[\Phi]} \delta\left(\Phi\left(-\Delta, x^{i}\right)-\Phi_{1}\left(x^{i}\right)\right) \\
& \cdot \delta\left(\Phi\left(\Delta, x^{i}\right)-\Phi_{2}\left(x^{i}\right)\right) .
\end{aligned}
$$

Note that since the path integral is performed over an $n$-sheeted Riemann surface, the action is now represented as $S_{n}[\Phi] . x_{1}^{l}, x_{2}^{l}$ represent the operator insersion points on the $l$-th sheet, and the boundary condition $\Phi_{1}, \Phi_{2}$ is now at the 1 -st and $n$-th Riemann sheet, respectively.

Thus, in the replica trick (Rényi) entanglement entropies for (2.4) and the ground state are respectively given by taking the trace $\log$ of $(2.7),{ }^{2}$

$$
\begin{aligned}
S_{A}^{(n), E X} & =\frac{1}{1-n} \log \left[\frac{\int D \Phi \mathcal{O}^{\dagger}\left(r_{1}, \theta_{1}^{n}\right) \mathcal{O}\left(r_{2}, \theta_{2}^{n}\right) \cdots \mathcal{O}\left(r_{1}, \theta_{1}^{1}\right)^{\dagger} \mathcal{O}\left(r_{2}, \theta_{2}^{1}\right) e^{-S_{n}[\Phi]}}{\left(\int D \Phi \mathcal{O}\left(r_{1}, \theta_{1}^{1}\right)^{\dagger} \mathcal{O}\left(r_{2}, \theta_{2}^{1}\right) e^{-S_{1}[\Phi]}\right)^{n}}\right] \\
S_{A}^{(n), G} & =\frac{1}{1-n} \log \left[\frac{\int D \Phi e^{-S_{n}[\Phi]}}{\left(\int D \Phi e^{-S_{1}[\Phi]}\right)^{n}}\right]
\end{aligned}
$$

where $\theta_{1,2}^{k}=\theta_{1,2}+2(k-1) \pi$. The actions $S_{n}$ and $S_{1}$ are defined on the n-sheeted geometry $\Sigma_{n}$ (see figure 3 ) and the flat space $\Sigma_{1}$, respectively. By substituting (Rényi) entanglement entropies in (2.8) into (2.3), $\Delta S_{A}^{(n)}$ is given by

$$
\Delta S_{A}^{(n)}=\frac{1}{1-n} \log \left[\frac{\left\langle\mathcal{O}^{\dagger}\left(r_{1}, \theta_{1}^{n}\right) \mathcal{O}\left(r_{2}, \theta_{2}^{n}\right) \cdots \mathcal{O}^{\dagger}\left(r_{1}, \theta_{1}^{1}\right) \mathcal{O}\left(r_{2}, \theta_{2}^{1}\right)\right\rangle_{\Sigma_{n}}}{\left\langle\mathcal{O}^{\dagger}\left(r_{1}, \theta_{1}^{1}\right) \mathcal{O}\left(r_{2}, \theta_{2}^{1}\right)\right\rangle_{\Sigma_{1}}^{n}}\right]
$$




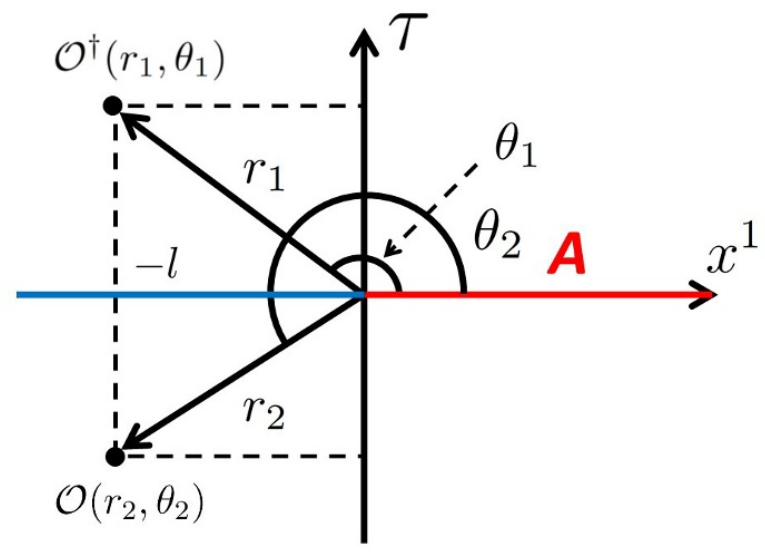

Figure 2. The location of local gauge invariant operator in Euclidean space.

We only need to compute propagators on $\Sigma_{n}$ in order to compute $\Delta S_{A}^{(n)}$ in free field theories. The two point function of gauge fields $A_{a}$ is defined by $-\left\langle A_{a}(r, \theta, \mathbf{x}) A_{b}\left(r^{\prime}, \theta^{\prime}, \mathbf{x}^{\prime}\right)\right\rangle=$ $G_{a b}\left(r, r^{\prime}, \theta-\theta^{\prime}, \mathbf{x}-\mathbf{x}^{\prime}\right)$. If we choose a specific gauge, ${ }^{3}$ their Green's functions obey the same equation of motion as that for $4 d$ free massless scalar field theory,

$$
\begin{aligned}
& \partial_{r}^{2} G_{b}^{a}\left(r, r^{\prime}, \theta-\theta^{\prime}, \mathbf{x}-\mathbf{x}^{\prime}\right)+\frac{1}{r} \partial_{r} G_{b}^{a}\left(r, r^{\prime}, \theta-\theta^{\prime}, \mathbf{x}-\mathbf{x}^{\prime}\right) \\
& +\frac{1}{r^{2}} \partial_{\theta}^{2} G_{b}^{a}\left(r, r^{\prime}, \theta-\theta^{\prime}, \mathbf{x}-\mathbf{x}^{\prime}\right)+\partial_{\mathbf{x}}^{2} G_{b}^{a}\left(r, r^{\prime}, \theta-\theta^{\prime}, \mathbf{x}-\mathbf{x}^{\prime}\right)=-\frac{\delta_{b}^{a} \delta\left(r-r^{\prime}\right) \delta\left(\theta-\theta^{\prime}\right) \delta^{2}\left(\mathbf{x}-\mathbf{x}^{\prime}\right)}{r},
\end{aligned}
$$

where $a=\left\{\tau, x^{1}, x^{2}, x^{3}\right\}{ }^{4}$

The solution of the equation is given by

$$
G_{a b}\left(r, r^{\prime}, \theta-\theta^{\prime}, \mathbf{x}-\mathbf{x}^{\prime}\right)=\frac{\delta_{a b} \sinh \left(\frac{t_{0}}{n}\right)}{8 n \pi^{2} r r^{\prime} \sinh t_{0}\left(\cosh \left(\frac{t_{0}}{n}\right)-\cos \left(\frac{\theta-\theta^{\prime}}{n}\right)\right)},
$$

where $t_{0}$ is defined by

$$
\cosh t_{0}=\frac{r^{2}+r^{\prime 2}+\left(x^{2}-x^{\prime 2}\right)^{2}+\left(x^{3}-x^{\prime 3}\right)^{2}}{2 r r^{\prime}} .
$$

(2.11) has been obtained by the authors in [44-46, 48, 60, 61].

Analytic continuation. After computing Green's functions on $\Sigma_{n}$ in Euclidean space, we perform the following analytic continuation,

$$
A_{\tau}=i A_{t}, \partial_{\tau}=i \partial_{t}, \tau_{l}=\epsilon-i t, \tau_{e}=-\epsilon-i t
$$

where $\epsilon$ is a smearing parameter which is introduced to keep the norm of the excited state finite. Analytic-continued Green's functions depend on $\epsilon$. We are interested in the behavior of $\Delta S_{A}^{(n)}$ in the limit $\epsilon \rightarrow 0$. Their leading behavior $\left(\sim \mathcal{O}\left(\frac{1}{\epsilon^{4}}\right)\right)$ are summarized in appendix A.

\footnotetext{
${ }^{2}$ This formula was introduced in [42, 43] . For detail of computation, see also [44-47].

${ }^{3}$ The chosen gauge corresponds to Feynman gauge in Minkowski spacetime.

${ }^{4} G_{b}^{a} \equiv \delta^{a c} G_{c b}$ where $\delta_{a c}=\operatorname{diag}(1,1,1,1)$.
} 


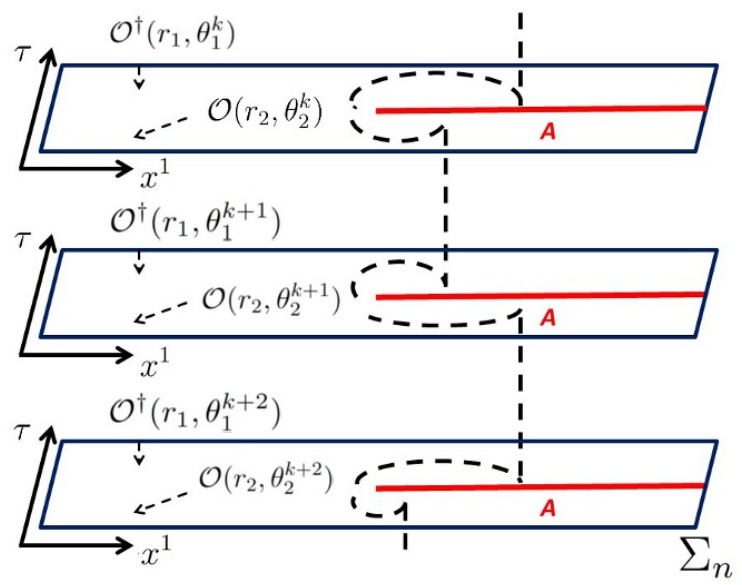

Figure 3. A picture of n-sheeted geometry $\Sigma_{n}$.

Gauge independence. The quantity $\Delta S_{A}^{(n)}$ does not depend on the choice of gauge fixing or boundary condition, since $\Delta S_{A}^{(n)}$ is the difference of the Rényi EE of ground state and excited state. More precisely, when we decompose the Hilbert space into two components, as discussed in [26], we need to specify a precise boundary condition. Here, we assume that this boundary condition can be imposed by an insertion of a corresponding local operator at the boundary. On the other hand, as is discussed in [45], the operator at the boundary does not contribute to the result since $\frac{l}{\epsilon} \gg 1$. Therefore, through out the computation of $\Delta S_{A}^{(n)}$, the result does not depend on the boundary condition. Moreover, we are allowed to choose an arbitrary boundary condition. Thus, we can set a gauge invariant boundary condition for the Green's functions of gauge invariant operators, so that the result will be gauge invariant.

\section{Excesses of (Rényi) entanglement entropy}

In this section, we study the time evolution and the late time value of $\Delta S_{A}^{(n)}$ in the following three cases.

(i) Only one electric or magnetic field $\mathcal{O}=E_{i}, B_{i}$ acts on the ground state. The time evolution of $\Delta S_{A}^{(n)}$ depends on the operator which acts on the ground state. If electromagnetic fields are changed by $F_{\mu \nu} \rightarrow \tilde{F_{\mu \nu}}=\frac{1}{2} \epsilon_{\mu \nu \rho \sigma} F^{\rho \sigma}, \Delta S_{A}^{(n)}$ does not change. Here $\epsilon_{\mu \nu \rho \sigma}$ is an antisymmetric tensor. The late-time value of $\Delta S_{A}^{(n)}$ does not depend on the operator. It can be interpreted in terms of the quasi-particle created by a scalar operator $\phi$.

(ii) Composite operators which act on the ground state are constructed of only electric or magnetic fields such as $\mathbf{E}^{2}$ and $\mathbf{B}^{2} . \Delta S_{A}^{(n)}$ for $\mathbf{E}^{2}$ is equivalent to the entropy for $\mathbf{B}^{2}$. Thus $\Delta S_{A}^{(n)}$ for them are invariant under the electric-magnetic duality transformation. There are no differences between their effect on the (Rényi) entanglement entropy. Its late-time values can be interpreted in terms of quasi-particles, which are created 
by the operator constructed of massless free scalar fields $\sum_{a=1}^{3}\left(\phi^{a}\right)^{2}$. Here $a$ denotes the kinds of fields.

(iii) Local operators are constructed of both electric and magnetic fields such as $E_{1}^{2}+B_{1}^{2}$, $B_{3} E_{2}$ and $\mathbf{B} \cdot \mathbf{E}$. The late-time value of $\Delta S_{A}^{(n)}$ shows that there is a significant difference between the effect of $E_{1}\left(B_{1}\right)$ and $E_{2,3}\left(B_{2,3}\right)$ on the late-time entanglement structure. Here $E_{1}\left(B_{1}\right)$ is the electric (magnetic) field along the direction vertical to the entangling surface. On the other hand, $E_{2,3}\left(B_{2,3}\right)$ is the electric (magnetic) field along the direction parallel to the entangling surface. As it will be explained in the next section, the difference comes from the commutation relation between electromagnetic quasi-particles created by $E_{2}\left(E_{3}\right)$ and the particles created by $B_{3}\left(B_{2}\right)$.

\section{$3.1 \mathcal{O}=E_{i}$ or $B_{i}$}

Here locally excited states are defined by acting with only $E_{i}$ or $B_{i}$ on the ground state. $\Delta S_{A}^{(n)}$ is given by (2.9) in the replica method with Euclidean signature. After performing the analytic continuation in (2.13) and taking the limit $\epsilon \rightarrow 0$, their time evolution is given as follows. $\Delta S_{A}^{(n)}$ vanishes before $t=l(>0)$, but after $t=l$, it increases. The detail of their time evolution is summarized in table 1. After taking the late time limit $(0<l \ll t)$, they are given by

$$
\Delta S_{A}^{(n)} \sim \log 2
$$

Their late time value is the same as that for $\phi$ in free massless scalar field theories with any spacetime dimensions. It can be interpreted as (Rényi) entanglement entropy for maximally entangled state in 2-qubit system. Therefore they do not show the difference between the effect of electromagnetic fields and that of free scalar one on the late-time structure of quantum entanglement. However, the time evolution of $\Delta S_{A}^{(n)}$ depends on the local operator which acts on the ground state as in figure 4 . Even at $t \sim l$, time evolution of $\Delta S_{A}^{(n)}$ depends on the one which acts on the ground state. If $\mathcal{O}=B_{2,3}$ or $E_{2,3}$ acts on the ground state, $\Delta S_{A}^{(n \geq 2)}$ is given by

$$
\Delta S_{A}^{(n)} \sim \frac{n}{n-1}\left(\frac{3(t-l)}{4 l}\right)+\cdots
$$

where $\cdots$ are contributions from the higher order $\mathcal{O}\left(\left(\frac{t-l}{l}\right)^{2}\right) . \Delta S_{A}^{(n \geq 2)}$ for $\mathcal{O}=E_{1}$ or $B_{1}$ at $t \sim l$ is given by

$$
\Delta S_{A}^{(n)} \sim \frac{n}{n-1}\left(\frac{3(t-l)^{2}}{4 l^{2}}\right)+\cdots .
$$

$\cdots$ are contributions from the higher order $\mathcal{O}\left(\left(\frac{t-l}{l}\right)^{3}\right)$. Their time evolution shows that quasi-particles created by $E_{2,3}\left(B_{2,3}\right)$ enter the region $A$ faster than those generated by $E_{1}\left(B_{1}\right)$. These behaviors seem to be natural since particles created by $E_{1}\left(B_{1}\right)$ do not propagate along the direction parallel to $x^{1}$.

$\Delta S_{A}^{(n)}$ in table 1 shows that they are invariant under the transformation,

$$
F_{\mu \nu} \rightarrow \frac{1}{2} \epsilon_{\mu \nu \rho \sigma} F^{\rho \sigma}
$$




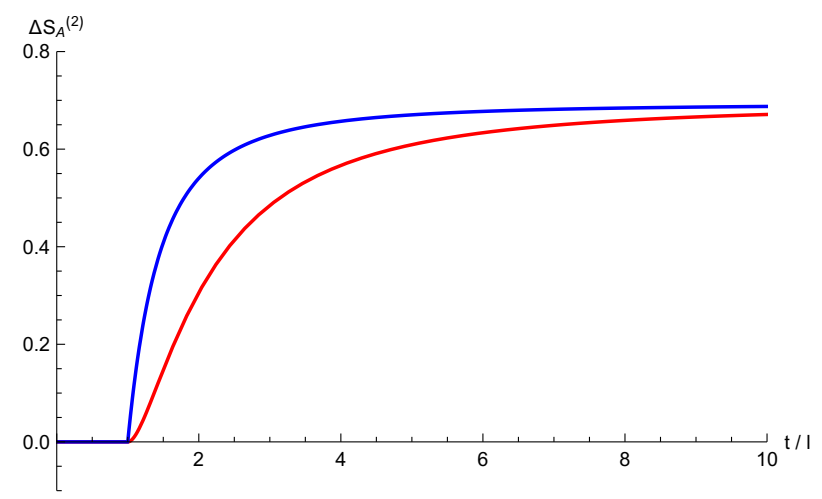

Figure 4. The time evolution of $\Delta S_{A}^{(2)}$ for $E_{1}\left(B_{1}\right)$ and $E_{2,3}\left(B_{2,3}\right)$. The horizontal and vertical axes correspond to time $t / l$ and $\Delta S_{A}^{(2)}$, respectively. The red and blue lines correspond to $\Delta S_{A}^{(2)}$ for $E_{1}\left(B_{1}\right)$ and $E_{2,3}\left(B_{2,3}\right)$, respectively.

\begin{tabular}{|l|c|}
\hline $\mathcal{O}$ & $\Delta S_{A}^{(n)}$ \\
\hline$E_{1}$ or $B_{1}$ & $\frac{1}{1-n} \log \left(\left(-\frac{(l+t)^{2}(l-2 t)}{4 t^{3}}\right)^{n}+\left(\frac{(t-l)^{2}(l+2 t)}{4 t^{3}}\right)^{n}\right)$ \\
\hline$E_{2,3}$ & $\frac{1}{1-n} \log \left(\left(\frac{-l^{3}-3 l t^{2}+4 t^{3}}{8 t^{3}}\right)^{n}+\left(\frac{l^{3}+3 l t^{2}+4 t^{3}}{8 t^{3}}\right)^{n}\right)$ \\
\hline
\end{tabular}

Table 1. $\Delta S_{A}^{(n)}$ for $\mathcal{O}$ in the region $0<l \leq t$.

where $\epsilon_{\mu \nu \rho \sigma}$ is an anti-symmetric tensor. Under the transformation in (3.4), the local operator $E_{i}\left(B_{i}\right)$ changes to $-B_{i}\left(E_{i}\right)$. Therefore, this duality changes a locally excited state to a different one.

\subsection{Composite operators constructed of only electric or magnetic fields}

The excited states which we consider here are generated by acting with the following operators: (a) $E_{i} E_{j}$ or $B_{i} B_{j}$, (b) $\mathbf{E}^{2}$ or $\mathbf{B}^{2}$. We study the time evolution and the late-time value of $\Delta S_{A}^{(n)}$ for them.

\subsection{1 $\mathcal{O}=E_{i} E_{j}$ or $B_{i} B_{j}$}

First we consider $\Delta S_{A}^{(n)}$ for the excited states generated by acting with $E_{i} E_{j}$ or $B_{i} B_{j}$ on the ground state. When $i=j$, the late time-value of $\Delta S_{A}^{(n)}$ is given by

$$
\Delta S_{A}^{(n)}=-\frac{1}{1-n} \log \frac{4^{n}}{2^{n}+2}
$$

It is the same as that of $\Delta S_{A}^{(n)}$ for $\phi^{2}$ in the massless free scalar field theories as in [44, 45]. Therefore, the late-time value of $\Delta S_{A}^{(n)}$ ((Rényi) entanglement entropy of operator) can be interpreted in terms of entangled quasi-particles created by $\phi^{2}$. 


\begin{tabular}{|l|l|l|l|l|}
\hline $\mathcal{O}$ & $D_{1}$ & $N_{1}$ & $N_{2}$ & $N_{3}$ \\
\hline$E_{1}^{2}$ or $B_{1}^{2}$ & $\left(2\left(\frac{1}{16}\right)^{2}\right)^{n}$ & $\left(2\left(f_{1}\right)^{2}\right)^{n}$ & $\left(2\left(f_{2}\right)^{2}\right)^{n}$ & $2^{2 n}\left(f_{1} f_{2}\right)^{n}$ \\
\hline$E_{2,3}^{2}$ or $B_{2,3}^{2}$ & $\left(2\left(\frac{1}{16}\right)^{2}\right)^{n}$ & $\left(2\left(f_{3}\right)^{2}\right)^{n}$ & $\left(2\left(f_{4}\right)^{2}\right)^{n}$ & $2^{2 n}\left(f_{3} f_{4}\right)^{n}$ \\
\hline $\begin{array}{l}E_{1} E_{2,3} \text { or } E_{1} B_{2,3} \\
\text { or } B_{1} E_{2,3} \text { or } B_{1} B_{2,3}\end{array}$ & $\left(\frac{1}{16}\right)^{2 n}$ & $\left(f_{1}\right)^{n}\left(f_{3}\right)^{n}$ & $\left(f_{2}\right)^{n}\left(f_{4}\right)^{n}$ & $\left(f_{2}\right)^{n}\left(f_{3}\right)^{n}+\left(f_{1}\right)^{n}\left(f_{4}\right)^{n}$ \\
\hline$E_{2} E_{3}$ or $B_{2} B_{3}$ & $\left(\frac{1}{16}\right)^{2 n}$ & $\left(f_{3}\right)^{2 n}$ & $\left(f_{4}\right)^{2 n}$ & $2\left(f_{3}\right)^{n}\left(f_{4}\right)^{n}$ \\
\hline$E_{2} B_{2}$ or $E_{3} B_{3}$ & $\left(\left(\frac{1}{16}\right)^{2}\right)^{n}$ & $\left(f_{3}\right)^{2 n}$ & $\left(f_{4}\right)^{2 n}$ & $2\left(f_{3}\right)^{n}\left(f_{4}\right)^{n}$ \\
\hline$E_{1} B_{1}$ & $\left(\frac{1}{16}\right)^{2 n}$ & $\left(f_{1}\right)^{2 n}$ & $\left(f_{2}\right)^{2 n}$ & $2\left(f_{1}\right)^{n}\left(f_{2}\right)^{n}$ \\
\hline Functions & $f_{1}=-\frac{(l-2 t)(l+t)^{2}}{64 t^{3}}$ & $f_{2}=\frac{(l+2 t)(l-t)^{2}}{64 t^{3}}$ & $f_{3}=\frac{l^{3}+3 l t^{2}+4 t^{3}}{128 t^{3}}$ & $f_{4}=\frac{-l^{3}-3 l t^{2}+4 t^{3}}{128 t^{3}}$ \\
\hline
\end{tabular}

Table 2. $\Delta S_{A}^{(n)}=\frac{1}{1-n} \log \left[\frac{N_{1}+N_{2}+N_{3}}{D_{1}}\right]$ for $\mathcal{O}$ in the region $0<l<t$.

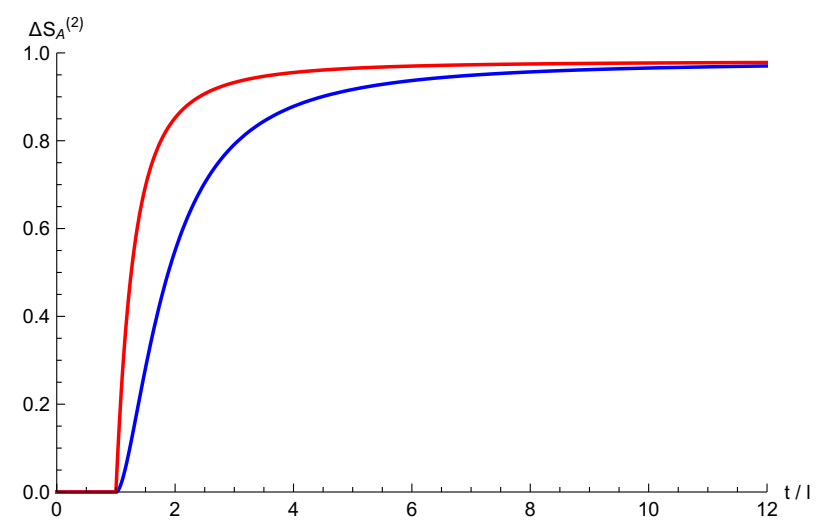

Figure 5. The time evolution of $\Delta S_{A}^{(2)}$ for $E_{1} E_{1}$ and $E_{2} E_{2}$. The horizontal and vertical axes correspond to time $t / l$ and the excess of $\Delta S_{A}^{(2)}$, respectively. The blue line represents the evolution for $E_{1} E_{1}$, and the red line for $E_{2} E_{2}$. In the limit $t \rightarrow \infty$, they are all $\log \frac{8}{3}$.

When $i \neq j, \Delta S_{A}^{(n)}$ at the late time is given by

$$
\Delta S_{A}^{(n)}=\log 4,
$$

which can be interpreted as maximum (Rényi) entanglement entropy for $\rho_{A}=$ $\frac{1}{4} \operatorname{diag}(1,1,1,1)$. It is the same as $\Delta S_{A}^{(n)}$ for the excited state given by acting with the operator $\phi^{a} \phi^{b}$ on the ground state. Here $a, b$ denote the kind of scalar fields, and $a \neq b$. There are two kinds of massless free scalar fields. The time evolution of $\Delta S_{A}^{(n)}$ for them is summarized in table 2. Table 2 shows $\Delta S_{A}^{(n)}$ is invariant under the transformation in (3.4).

The time dependence of each excitation is displayed in figures 5 and 6 . The evolutions without taking the limit $t \rightarrow \infty$ is different. However, amazingly the $\Delta S_{A}^{(2)}$ agree in the limit $t \rightarrow \infty$, for each cases. 


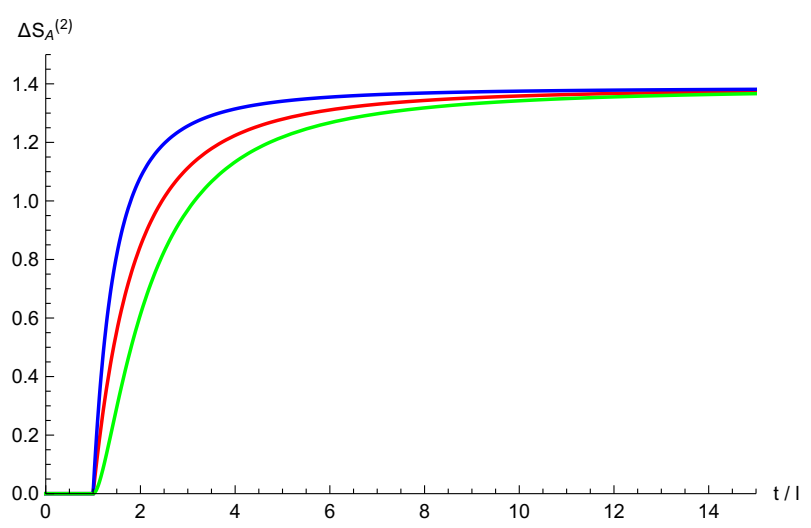

Figure 6. The time evolution of $\Delta S_{A}^{(2)}$ for $E_{1} E_{2}, E_{2} E_{3}\left(E_{2} B_{2}\right)$ and $E_{1} B_{1}$. The horizontal and vertical axes correspond to time $t / l$ and the excess of $\Delta S_{A}^{(2)}$, respectively. The red line represents the evolution for $E_{1} E_{2}$, the blue line for $E_{2} E_{3}\left(E_{2} B_{2}\right)$ and the green line for $E_{1} B_{1}$. In the limit $t \rightarrow \infty$, they are all $2 \log 2$.

\subsection{2 $\mathcal{O}=\mathrm{E}^{2}$ or $\mathrm{B}^{2}$}

In order to study whether $E_{1}$ acts on the late-time structure of quantum entanglement differently from $E_{2,3},{ }^{5}$ we study the late-time value of $\Delta S_{A}^{(n)}$ for the given locally excited state:

$$
|\Psi\rangle=\mathcal{N} \mathbf{E}^{2}(-t,-l, \mathbf{x})|0\rangle
$$

Before studying its late-time value, we comment on its time evolution. Before $t=l, \Delta S_{A}^{(n)}$ for the state in (3.7) vanishes and after $t=l$, it increases. Its time evolution is summarized in table 3 .

After $t=l$, as in table $3, \Delta S_{A}^{(n)}$ is given by

$$
\Delta S_{A}^{(n)}=\frac{1}{1-n} \log \left[\frac{N_{1}+N_{2}+P_{1}+P_{2}+P_{3}}{D}\right],
$$

where $D, N_{i}$ and $P_{i}$ are defined in table 3 . If we take the late time limit $(0<l \ll t)$, the ratios of $P_{i}$ and $N_{i}$ to $D$ reduce to constant numbers [62],

$$
\left(\frac{N_{1}}{D}\right)^{\frac{1}{n}}=\left(\frac{N_{2}}{D}\right)^{\frac{1}{n}}=4^{-1}, \quad\left(\frac{P_{1}}{D}\right)^{\frac{1}{n}}=\left(\frac{P_{2}}{D}\right)^{\frac{1}{n}}=\left(\frac{P_{3}}{D}\right)^{\frac{1}{n}}=\left(\frac{1}{6}\right)
$$

where we ignore the higher order contribution $\mathcal{O}\left(\frac{l}{t}\right)$. Amazingly, the sum of them is 1 . Therefore if the effective reduced density matrix is defined by

$$
\Delta S_{A}^{(n)}=\frac{1}{1-n} \log \left[\operatorname{tr}_{A}\left(\rho_{A}^{e}\right)^{n}\right]
$$

then the matrix is given by

$$
\rho_{A}^{e}=\frac{1}{24} \operatorname{diag}(6,4,4,4,6)
$$

\footnotetext{
${ }^{5}$ The effect of $E_{1}$ can be different from that of $E_{2,3}$ on the structure since we choose $t=0, x^{1} \geq 0$ as the subsystem $A$.
} 


\begin{tabular}{|l|l|l|l|l|l|l|}
\hline $\mathcal{O}$ & $D_{1}$ & $N_{1}$ & $N_{2}$ & $P_{1}$ & $P_{2}$ & $P_{3}$ \\
\hline $\mathbf{B}^{2}$ or $\mathbf{E}^{2}$ & $\left(2 \cdot 3\left(\frac{1}{16}\right)^{2}\right)^{n}$ & $\left(2 f_{1}^{2}+2 \cdot 2 f_{3}^{2}\right)^{n}$ & $\left(2 f_{2}^{2}+2 \cdot 2 f_{4}^{2}\right)^{n}$ & $2^{2 n} f_{1}^{n} f_{2}^{n}$ & $2^{2 n} f_{3}^{n} f_{4}^{n}$ & $2^{2 n} f_{3}^{n} f_{4}^{n}$ \\
\hline$B_{1}^{2}+E_{1}^{2}$ & $\left(2 \cdot 2\left(\frac{1}{16}\right)^{2}\right)^{n}$ & $\left(2 \cdot 2 f_{1}^{2}\right)^{n}$ & $\left(2 \cdot 2 f_{2}^{2}\right)^{n}$ & $2^{2 n} f_{1}^{n} f_{2}^{n}$ & $2^{2 n} f_{1}^{n} f_{2}^{n}$ & 0 \\
\hline $\begin{array}{l}E_{2} B_{3} \\
\text { or } E_{3} B_{2}\end{array}$ & $\left(2^{2}\left(\frac{1}{4 \cdot 8}\right)^{2}\right)^{n}$ & $\left(2\left(g_{1}\right)^{2}+2\left(g_{3}\right)^{2}\right)^{n}$ & $\left(2\left(g_{2}\right)^{2}+2\left(g_{4}\right)^{2}\right)^{n}$ & $2^{2 n}\left(g_{2}\right)^{n}\left(g_{3}\right)^{n}$ & $2^{2 n}\left(g_{1}\right)^{n}\left(g_{4}\right)^{n}$ & 0 \\
\hline $\begin{array}{l}F^{\mu \nu} F_{\mu \nu} \\
\text { or } \mathbf{B} \cdot \mathbf{E}\end{array}$ & $\left(2 \cdot 2\left(\frac{1}{16}\right)^{2}+2 \cdot 4^{2}\left(\frac{1}{4 \cdot 8}\right)^{2}\right)^{n}$ & $\left(2 \cdot 2 f_{1}^{2}+2 \cdot 4^{2} g_{1} \cdot g_{3}\right)^{n}$ & $\left(2 \cdot 2 f_{2}^{2}+2 \cdot 4^{2} g_{2} \cdot g_{4}\right)^{n}$ & $2 \cdot 2^{2 n}\left(f_{1}\right)^{n}\left(f_{2}\right)^{n}$ & $2 \cdot 4^{2 n}\left(g_{1}\right)^{n}\left(g_{2}\right)^{n}$ & $2 \cdot 4^{2 n}\left(g_{3}\right)^{n}\left(g_{4}\right)^{n}$ \\
\hline$B_{2} E_{3}-B_{3} E_{2}$ & $\left(4 \cdot 2\left(\frac{1}{4 \cdot 8}\right)^{2}\right)^{n}$ & $\left(2 \cdot 2 g_{3}^{2}+2 \cdot 2 g_{1}^{2}\right)^{n}$ & $\left(2 \cdot 2 g_{4}^{2}+2 \cdot 2 g_{2}^{2}\right)^{n}$ & $2^{2 n}\left(g_{3}\right)^{n}\left(g_{2}\right)^{n}$ & $2^{2 n}\left(g_{1}\right)^{n}\left(g_{4}\right)^{n}$ & 0 \\
\hline Functions & $g_{1}=\frac{(l+t)^{3}}{4 \cdot 64 t^{3}}$ & $g_{2}=\frac{(t-l)^{3}}{4 \cdot 64 t^{3}}$ & $g_{3}=\frac{(l+t)\left(l^{2}-4 l t+7 t^{2}\right)}{4 \cdot 64 t^{3}}$ & $g_{4}=\frac{(t-l)\left(l^{2}+4 l t+7 t^{2}\right)}{4 \cdot 64 t^{3}}$ & & \\
\hline
\end{tabular}

Table 3. $\Delta S_{A}^{(n)}=\frac{1}{1-n} \log \left[\frac{N_{1}+N_{2}+P_{1}+P_{2}+P_{3}}{D_{1}}\right]$ for $\mathcal{O}$ in the region $0<l<t$.

The excess of $n$-th (Rényi) entanglement entropy, entanglement entropy and Min entropy are respectively given by

$$
\begin{aligned}
\Delta S_{A}^{(n)} & =\frac{1}{n-1} \log \left(\frac{12^{n}}{3 \cdot 2^{n}+2 \cdot 3^{n}}\right) \\
\Delta S_{A} & =\frac{\log (24)}{2} \\
\Delta S_{A}^{(\infty)} & =\log 4
\end{aligned}
$$

which can be interpreted in terms of quasi-particles created by $\left(\phi^{1}\right)^{2}+\left(\phi^{2}\right)^{2}+\left(\phi^{3}\right)^{2}$, which is constructed of three kinds of free scalar fields. Therefore, there are no differences between the effect of $E_{1}$ and that of $E_{2,3}$ on the late-time structure of quantum entanglement. As in the table 2, $\Delta S_{A}^{(n)}$ for $\mathbf{E}^{2}$ is equivalent to that for $\mathbf{B}^{2}$. Therefore, they are electric-magnetic duality invariants.

\subsection{Composite operators constructed of both electric and magnetic fields}

In the previous two subsections we study how the entanglement structure changes at the late time if either electric or magnetic fields act on the ground state. However, we do not uncover how it changes at the late time when both of them act on the ground state. Here we study $\Delta S_{A}^{(n)}$ for (a) $E_{1}^{2}+B_{1}^{2}$, (b) $E_{i} B_{j}$ and (c) $F^{\mu \nu} F_{\mu \nu}$ and $\mathbf{B} \cdot \mathbf{E}$, which can show that $E_{i}$ and $B_{i}$ act on the late-time structure of quantum entanglement differently from scalar fields such as $\phi^{a}$.

\subsection{1 $\quad E_{1}^{2}+B_{1}^{2}$}

Here in order to study whether there are differences between the effects of electric and magnetic fields on the late-time structure of quantum entanglement, we study $\Delta S_{A}^{(n)}$ for the following excited state:

$$
|\Psi\rangle=\mathcal{N}\left(E_{1}^{2}+B_{1}^{2}\right)(-t,-l, \mathbf{x})|0\rangle .
$$

Before investing the late time value of $\Delta S_{A}^{(n)}$, let's study the time evolution of $\Delta S_{A}^{(n)}$. $\Delta S_{A}^{(n)}$ vanishes before $t=l$. After $t=l$, its time evolution is summarized in table 3. If 
we take the late time limit $t \rightarrow \infty$, the late time value of $\Delta S_{A}^{(n)}$ reduces to the (Rényi) entanglement entropy whose effective reduced density matrix is given by

$$
\rho_{A}^{e}=\frac{1}{4} \operatorname{diag}(1,1,1,1) .
$$

Its entropies are given by

$$
\Delta S_{A}^{(n)}=\Delta S_{A}=\Delta S_{A}^{(\infty)}=\log 4
$$

It shows there are no differences between the effects of electric and magnetic fields on the late-time structure.

\subsection{2 $\quad E_{i} B_{j}$}

Here let's find out how the operators constructed of both electric and magnetic fields, $E_{i} B_{j}$, affect the late-time structure of quantum entanglement. The late-time values of $\Delta S_{A}^{(n)}$ for $E_{i} B_{j}$ except for $E_{2} B_{3}$ and $E_{3} B_{2}$ are the same as (3.6). Their time evolution is summarized in table 2 .

On the other hand, after $t=l$ the time evolution of $\Delta S_{A}^{(n)}$ for $E_{2} B_{3}$ or $E_{3} B_{2}$ is summarized in table 3 . We can see that it has the electric-magnetic duality from the table 3. The late-time value of $\Delta S_{A}^{(n)}$ is given by (Rényi) entanglement entropy whose reduced density matrix is given by

$$
\rho_{A}^{e}=\frac{1}{64} \operatorname{diag}(25,7,7,25) .
$$

Its entropies are given by

$$
\begin{aligned}
\Delta S_{A}^{(n)} & =-\frac{\log \left(2^{1-6 n}\left(7^{n}+25^{n}\right)\right)}{n-1}, \\
\Delta S_{A} & =\log \left(\frac{64}{5 \cdot 5^{9 / 16} 7^{7 / 32}}\right), \\
\Delta S_{A}^{(\infty)} & =2 \log \left(\frac{8}{5}\right) .
\end{aligned}
$$

It shows how different the effect of $E_{1}\left(B_{1}\right)$ is from that of $E_{2,3}\left(B_{2,3}\right)$ on the structure. The value can not be interpreted in terms of quasi-particles created by scalar fields such as $\phi^{a} \phi^{b}$. As we will explain later, in the entangled quasi-particle interpretation, there is a commutation relation between the quasi-particle created by $E_{2}\left(B_{2}\right)$ and that by $B_{3}\left(E_{3}\right)$.

\subsubsection{B $\cdot \mathrm{E}$ and $F_{\mu \nu} F^{\mu \nu}$ and $B_{2} E_{3}-B_{3} E_{2}$}

We finally study $\Delta S_{A}^{(n)}$ for more complicated operators, B $\cdot \mathbf{E}, F_{\mu \nu} F^{\mu \nu}$ and $B_{2} E_{3}-B_{3} E_{2}$. Before $t=l, \Delta S_{A}^{(n)}$ for them vanishes, but after $t=l$, it increases. Their details are summarized in table $3 .^{6}$ It shows that $\Delta S_{A}^{(n)}$ for $\mathbf{B} \cdot \mathbf{E}$ is the same as that for $F^{\mu \nu} F_{\mu \nu}$.

${ }^{6} \Delta S_{A}^{(n)}$ is commuted by the Green's functions in appendix B. 
The effective reduced density matrices for $\mathbf{B} \cdot \mathbf{E}\left(F^{\mu \nu} F_{\mu \nu}\right)$ and $B_{2} E_{3}-B_{3} E_{2}$ are given by

$$
\begin{array}{ll}
\rho_{A}^{e}=\frac{1}{192} \operatorname{diag}(30,30,16,16,49,49,1,1), & \text { for } \mathcal{O}=\mathbf{B} \cdot \mathbf{E}\left(F^{\mu \nu} F_{\mu \nu}\right), \\
\rho_{A}^{e}=\frac{1}{128} \operatorname{diag}(50,50,7,7,7,7), & \text { for } \mathcal{O}=B_{2} E_{3}-B_{3} E_{2} .
\end{array}
$$

Entropies are respectively given by

$$
\begin{aligned}
\Delta S_{A}^{(n)} & =\frac{1}{n-1} \log \left(\frac{2^{6 n-1} 3^{n}}{16^{n}+30^{n}+49^{n}+1}\right), \\
\Delta S_{A} & =\log \left(\frac{32 \cdot 3^{11 / 16 \sqrt[48]{\frac{2}{7}}}}{7 \cdot 5^{5 / 16}}\right), \\
\Delta S_{A}^{(\infty)} & =\log \left(\frac{192}{49}\right) .
\end{aligned}
$$

which are for $\mathcal{O}=\mathbf{B} \cdot \mathbf{E}\left(\right.$ or $\left.F^{\mu \nu} F_{\mu \nu}\right)$, and

$$
\begin{aligned}
\Delta S_{A}^{(n)} & =-\frac{\log \left(2^{1-7 n}\left(2 \cdot 7^{n}+50^{n}\right)\right)}{n-1}, \\
\Delta S_{A} & =\log \left(\frac{64\left(\frac{2}{7}\right)^{7 / 32}}{5 \cdot 5^{9 / 16}}\right) \\
\Delta S_{A}^{(\infty)} & =2 \log \left(\frac{8}{5}\right)
\end{aligned}
$$

which are for $\mathcal{O}=B_{2} E_{3}-B_{3} E_{2}$. As we will explain in the next section, they can be reproduced by using a late-time algebra which electromagnetic quasi-particles obey.

The time dependence of $\mathbf{E}^{2}, F^{\mu \nu} F_{\mu \nu}$ and $B_{2} E_{3}-B_{3} E_{2}$ are shown in figure 7 . We can see that their $\Delta S_{A}^{(2)}$ increases diffently and reach different finite values in the limit $t \rightarrow \infty$. As we see in the following section, we find significant differences in the late time algebra between $\mathbf{E}^{2}$ and the others.

\section{A late-time algebra}

We interpret the late-time value of $\Delta S_{A}^{(n)}$ in terms of quasi-particles. More precisely, let's interpret the effective reduced density matrix in (3.10) in terms of quasi-particles. The effective reduced density matrix for the excited state generated by a composite operator $\mathcal{O}(-t,-l, \mathbf{x})$ is defined by

$$
\Delta S_{A}^{(n)}=\frac{1}{1-n} \log \left[\operatorname{tr}_{A}\left(\rho_{A}^{e}\right)^{n}\right]=\frac{1}{1-n} \log \left[\operatorname{tr}_{A}\left(\hat{\mathcal{N}}^{2} \mathcal{O}|0\rangle\langle 0| \mathcal{O}^{\dagger}\right)^{n}\right],
$$

where $\hat{\mathcal{N}}$ is a normalization constant. The operator $\mathcal{O}$ is assumed to be constructed of electric and magnetic fields. ${ }^{7}$ As in [44-46, 48], these fields can be decomposed into left

\footnotetext{
${ }^{7}$ Here $\rho_{A}^{e}$ is not the same as the reduced density matrix for the locally excited state. It is for a "effective" state $\hat{\mathcal{N O}}|0\rangle$. It is different form the "original" locally excited state.
} 


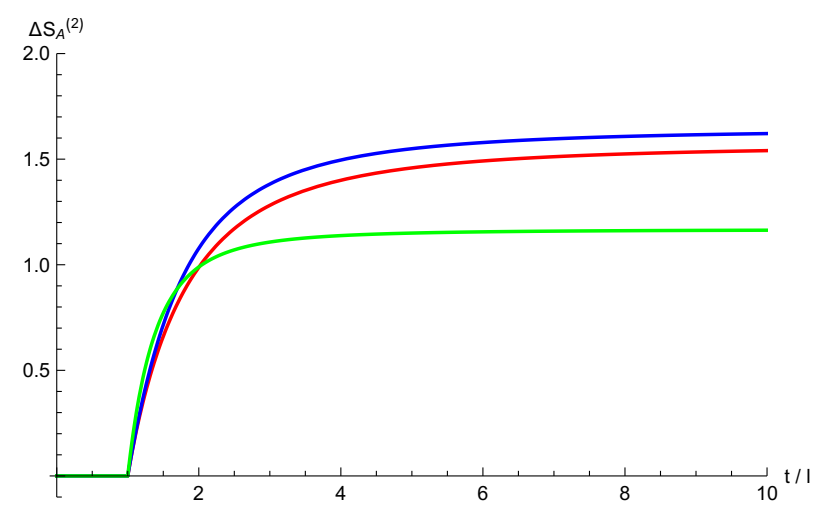

Figure 7. The time evolution of $\mathbf{E}^{2}, F^{\mu \nu} F_{\mu \nu}$ and $B_{2} E_{3}-B_{3} E_{2}$. The horizontal and vertical axes correspond to time $t / l$ and the excess of $\Delta S_{A}^{(2)}$ respectively. The red line represents the evolution for $\mathbf{E}^{2}$, the blue line for $F^{\mu \nu} F_{\mu \nu}$ and the green line for $B_{2} E_{3}-B_{3} E_{2}$.

moving and right moving electromagnetic quasi-particles as follows,

$$
\begin{aligned}
& E_{i}=E_{i}^{L \dagger}+E_{i}^{R \dagger}+E_{i}^{L}+E_{i}^{R}, \\
& B_{i}=B_{i}^{L \dagger}+B_{i}^{R \dagger}+B_{i}^{L}+B_{i}^{R},
\end{aligned}
$$

where since we take $x^{1} \geq 0$ as $A$ in this paper, left-moving and right-moving quasi-particles correspond to particles included in $B$ and $A$ at late time, respectively. The ground state for them is defined by

$$
\begin{aligned}
E_{i}^{L, R}|0\rangle_{L, R} & =B_{i}^{L, R}|0\rangle_{L, R}=0, \\
|0\rangle & =|0\rangle_{L} \otimes|0\rangle_{R} .
\end{aligned}
$$

The late-time algebra which quasi-particles obey is given by

$$
\begin{aligned}
& {\left[E_{i}^{L, R}, E_{j}^{L, R \dagger}\right]=C \delta_{i j},} \\
& {\left[B_{i}^{L, R}, B_{j}^{L, R \dagger}\right]=C \delta_{i j},}
\end{aligned}
$$

which is obtained so that the results by the replica trick are reproduced. Here $C$ is a real number. ${ }^{8}$ In the gauge theory in addition to (4.2), we need the following commutation relation for different particles:

$$
\left[E_{3}^{L, R}, B_{2}^{L, R \dagger}\right]=X_{R, L}, \quad\left[E_{2}^{L, R}, B_{3}^{L, R \dagger}\right]=Y_{R, L},
$$

where $X_{R, L}$ and $Y_{R, L}$ are given by

$$
\begin{aligned}
X_{R} & =-X_{L}=Y_{L}=-Y_{R}, \\
X_{R, L}^{2} & =Y_{R, L}^{2}=\frac{9}{16} C^{2} .
\end{aligned}
$$

\footnotetext{
${ }^{8}$ The redefinition of quasi-particles can absorb the constant $C$.
} 
Here $X_{R, L}$ and $Y_{R, L}$ are real numbers. ${ }^{9}$ The commutation relation between electric (magnetic) quasi-particles is determined so that the effective density matrices computed by (4.4) are consistent with (3.11) respectively. The relation for the quasi-particles of $E_{1}$ should be the same as that for $B_{1}$ so that the effective density matrix in (4.1) reproduces $\Delta S_{A}^{(n)}$ for the matrix in (3.14). That relation between quasi-particles generated by $E_{2}\left(E_{3}\right)$ and those by $B_{3}\left(B_{2}\right)$ reproduces the matrix in (3.16). We also check that $\Delta S_{A}^{(n)}$ for $\mathcal{O}=\mathbf{B} \cdot \mathbf{E}\left(F^{\mu \nu} F_{\mu \nu}\right)$, $B_{3} E_{2}-B_{2} E_{3}$ are reproduced by using the commutation relation in (4.4) and (4.5).

The relation in (4.5) shows that the effect of fields along the direction vertical to $\partial A$ is significantly different from that along the direction parallel to $\partial A$ on the late-time structure. It makes the effects of electromagnetic fields different from that of free scalar fields on the late-time structure of quantum entanglement.

\section{Conclusion and future problems}

We have studied how gauge invariant operators such as $E_{i}, B_{i}$ and the composite operators constructed of them changes the structure of quantum entanglement by studying $\Delta S_{A}^{(n)}$. We studied whether $\Delta S_{A}^{(n)}$ for locally excited states created by gauge invariant local operators reflects the electric-magnetic duality. $\Delta S_{A}^{(n)}$, which we studied in this paper, is invariant under the duality transformation. If only $E_{i}$ or $B_{i}$ acts on the ground state, without taking the late time limit, the time evolution of $\Delta S_{A}^{(n)}$ depends on them. Due to the duality, $\Delta S_{A}^{(n)}$ for $E_{i}$ is equal to that for $B_{i}$. Around $t=l, \Delta S_{A}^{(n)}$ for $E_{2,3}\left(B_{2,3}\right)$ increases slower than that for $E_{1}\left(B_{1}\right)$. However they can not show the difference between the effects of electromagnetic fields and that of scalar fields on the late-time structure because the late-time values of $\Delta S_{A}^{(n)}$ for them can be interpreted in terms of quasi-particle created by scalar fields.

On the other hand, the late-time values of $\Delta S_{A}^{(n)}$ for the specific operators constructed of both electric and magnetic fields can not be interpreted in terms of quasi-particles by scalar fields. They show that there are differences between the effects of electromagnetic fields and that of scalar fields on the late-time structure of quantum entanglement. If their late-time values are interpreted in terms of electromagnetic quasi-particles in (4.2), there are commutation relations between $E_{2}\left(E_{3}\right)$ and $B_{3}\left(B_{2}\right)$, which make the effect of electromagnetic field significantly different from that of scalar fields on the late-time structure. The effect of $E_{1}$ and $B_{1}$ on the late-time structure is different from that of $E_{2,3}$ and $B_{2,3}$.

Future problems. We finish with comments on a few future problems:

- In this paper we only consider $4 d$ Maxwell gauge theory which has conformal symmetry. $D(\neq 4)$ dimensional Maxwell gauge theory is not a CFT. Therefore, it is interesting to generalize the analysis in $4 d$ Maxwell theory to that in the theories with general dimensions.

\footnotetext{
${ }^{9}$ We find the correspondence between propagators and commutation relations. The commutations can be defined by the late time limit of propagators. We will discuss the detail of the correspondence in [62]. When we use this correspondence, $X_{L}=-\frac{3}{4} C$.
} 
- We expect that the structure of the late-time algebra depends on the spacetime dimension $D$. Then, it is also interesting to study it in general dimensions.

\section{Acknowledgments}

MN thanks Tadashi Takayanagi for useful discussions and comments on this paper. MN and NW thank Pawel Caputa, Tokiro Numasawa, Shunji Matsuura and Akinori Tanaka for useful comments on this work.

\section{A Green's functions}

The relation between $E_{i}, B_{i}$ and field strengths which are defined in Euclidean space is given by

$$
E_{i}=-i F_{\tau i}, \quad B_{1}=-F_{23}, \quad B_{2}=F_{13}, \quad B_{3}=-F_{12} .
$$

The analytic continued Green's functions are defined by

$$
\begin{aligned}
& \left\langle E_{1}(\theta) E_{1}\left(\theta^{\prime}\right)\right\rangle=F_{E 1 E 1}\left(\theta-\theta^{\prime}\right), \\
\left\langle E_{2}(\theta) E_{2}\left(\theta^{\prime}\right)\right\rangle= & \left\langle E_{3}(\theta) E_{3}\left(\theta^{\prime}\right)\right\rangle=F_{E 2 E 2}\left(\theta-\theta^{\prime}\right), \\
& \left\langle B_{1}(\theta) B_{1}\left(\theta^{\prime}\right)\right\rangle=F_{B 1 B 1}\left(\theta-\theta^{\prime}\right), \\
\left\langle B_{2}(\theta) B_{2}\left(\theta^{\prime}\right)\right\rangle= & \left\langle B_{3}(\theta) B_{3}\left(\theta^{\prime}\right)\right\rangle=F_{B 2 B 2}\left(\theta-\theta^{\prime}\right), \\
& \left\langle E_{2}(\theta) B_{3}\left(\theta^{\prime}\right)\right\rangle=F_{E 2 B 3}\left(\theta-\theta^{\prime}\right), \\
& \left\langle B_{3}(\theta) E_{2}\left(\theta^{\prime}\right)\right\rangle=F_{B 3 E 2}\left(\theta-\theta^{\prime}\right), \\
& \left\langle E_{3}(\theta) B_{2}\left(\theta^{\prime}\right)\right\rangle=F_{E 3 B 2}\left(\theta-\theta^{\prime}\right), \\
& \left\langle B_{2}(\theta) E_{3}\left(\theta^{\prime}\right)\right\rangle=F_{B 2 E 3}\left(\theta-\theta^{\prime}\right) .
\end{aligned}
$$

If the limit $\epsilon \rightarrow 0$ is taken, their leading terms for $n=1$ are given by

$$
\begin{aligned}
F_{E 1 E 1}\left(\theta_{1}-\theta_{2}\right) & \sim \frac{1}{16 \pi^{2} \epsilon^{4}}, \\
F_{E 2 E 2}\left(\theta_{1}-\theta_{2}\right) & \sim \frac{1}{16 \pi^{2} \epsilon^{4}}, \\
F_{B 1 B 1}\left(\theta_{1}-\theta_{2}\right) & \sim \frac{1}{16 \pi^{2} \epsilon^{4}}, \\
F_{B 2 B 2}\left(\theta_{1}-\theta_{2}\right) & \sim \frac{1}{16 \pi^{2} \epsilon^{4}} .
\end{aligned}
$$

That for the arbitrary $n$ in $0<t<l$ are the same as given in (A.3). 
That for the arbitrary $n$ in $0<l \leq t$ are given by

$$
\begin{aligned}
& F_{E 1 E 1}\left(\theta_{1}-\theta_{2}\right)=F_{E 1 E 1}\left(\theta_{2}-\theta_{1}\right) \sim-\frac{(l-2 t)(l+t)^{2}}{64 \pi^{2} t^{3} \epsilon^{4}}, \\
& F_{E 2 E 2}\left(\theta_{1}-\theta_{2}\right)=F_{E 2 E 2}\left(\theta_{2}-\theta_{1}\right) \sim \frac{l^{3}+3 l t^{2}+4 t^{3}}{128 \pi^{2} t^{3} \epsilon^{4}}, \\
& F_{B 1 B 1}\left(\theta_{1}-\theta_{2}\right)=F_{B 1 B 1}\left(\theta_{2}-\theta_{1}\right) \sim-\frac{(l-2 t)(l+t)^{2}}{64 \pi^{2} t^{3} \epsilon^{4}}, \\
& F_{B 2 B 2}\left(\theta_{1}-\theta_{2}\right)=F_{B 2 B 2}\left(\theta_{2}-\theta_{1}\right) \sim \frac{l^{3}+3 l t^{2}+4 t^{3}}{128 \pi^{2} t^{3} \epsilon^{4}}, \\
& F_{E 2 B 3}\left(\theta_{1}-\theta_{2}\right)=F_{E 2 B 3}\left(\theta_{2}-\theta_{1}\right) \sim \frac{3(t-l)(l+t)}{128 \pi^{2} t^{2} \epsilon^{4}}, \\
& F_{B 3 E 2}\left(\theta_{1}-\theta_{2}\right)=F_{B 3 E 2}\left(\theta_{2}-\theta_{1}\right) \sim \frac{3(t-l)(l+t)}{128 \pi^{2} t^{2} \epsilon^{4}}, \\
& F_{E 3 B 2}\left(\theta_{1}-\theta_{2}\right)=F_{E 3 B 2}\left(\theta_{2}-\theta_{1}\right) \sim \frac{3(l-t)(l+t)}{128 \pi^{2} t^{2} \epsilon^{4}}, \\
& F_{B 2 E 3}\left(\theta_{1}-\theta_{2}\right)=F_{B 2 E 3}\left(\theta_{2}-\theta_{1}\right) \sim \frac{3(l-t)(l+t)}{128 \pi^{2} t^{2} \epsilon^{4}}, \\
& F_{E 1 E 1}\left(\theta_{1}-\theta_{2}+2 \pi\right)=F_{E 1 E 1}\left(\theta_{2}-\theta_{1}-2 \pi\right) \\
& =F_{E 1 E 1}\left(\theta_{1}-\theta_{2}-2(n-1) \pi\right)=F_{E 1 E 1}\left(\theta_{2}-\theta_{1}+2(n-1) \pi\right) \sim \frac{(l-t)^{2}(l+2 t)}{64 \pi^{2} t^{3} \epsilon^{4}}, \\
& F_{E 2 E 2}\left(\theta_{1}-\theta_{2}+2 \pi\right)=F_{E 2 E 2}\left(\theta_{2}-\theta_{1}-2 \pi\right) \\
& =F_{E 2 E 2}\left(\theta_{1}-\theta_{2}-2(n-1) \pi\right)=F_{E 2 E 2}\left(\theta_{2}-\theta_{1}+2(n-1) \pi\right) \sim \frac{l^{3}+3 l t^{2}-4 t^{3}}{128 \pi^{2} t^{3} \epsilon^{4}} \text {, } \\
& F_{B 1 B 1}\left(\theta_{1}-\theta_{2}+2 \pi\right)=F_{B 1 B 1}\left(\theta_{2}-\theta_{1}-2 \pi\right) \\
& =F_{B 1 B 1}\left(\theta_{1}-\theta_{2}-2(n-1) \pi\right)=F_{B 1 B 1}\left(\theta_{2}-\theta_{1}+2(n-1) \pi\right) \sim \frac{(l-t)^{2}(l+2 t)}{64 \pi^{2} t^{3} \epsilon^{4}} \text {, } \\
& F_{B 2 B 2}\left(\theta_{1}-\theta_{2}+2 \pi\right)=F_{B 2 B 2}^{(n, l)}\left(\theta_{2}-\theta_{1}-2 \pi\right) \\
& =F_{B 2 B 2}\left(\theta_{1}-\theta_{2}-2(n-1) \pi\right)=F_{B 2 B 2}^{(n, l)}\left(\theta_{2}-\theta_{1}+2(n-1) \pi\right) \sim \frac{l^{3}+3 l t^{2}-4 t^{3}}{128 \pi^{2} t^{3} \epsilon^{4}} \text {, } \\
& F_{E 2 B 3}\left(\theta_{1}-\theta_{2}+2 \pi\right)=F_{E 2 B 3}\left(\theta_{2}-\theta_{1}-2 \pi\right) \\
& =F_{E 2 B 3}\left(\theta_{1}-\theta_{2}-2(n-1) \pi\right)=F_{E 2 B 3}\left(\theta_{2}-\theta_{1}+2(n-1) \pi\right) \sim \frac{3(l-t)(l+t)}{128 \pi^{2} t^{2} \epsilon^{4}} \text {, } \\
& F_{B 3 E 2}\left(\theta_{1}-\theta_{2}+2 \pi\right)=F_{B 3 E 2}\left(\theta_{2}-\theta_{1}-2 \pi\right) \\
& =F_{B 3 E 2}\left(\theta_{1}-\theta_{2}-2(n-1) \pi\right)=F_{B 3 E 2}\left(\theta_{2}-\theta_{1}+2(n-1) \pi\right) \sim \frac{3(l-t)(l+t)}{128 \pi^{2} t^{2} \epsilon^{4}}, \\
& F_{E 3 B 2}\left(\theta_{1}-\theta_{2}+2 \pi\right)=F_{E 3 B 2}\left(\theta_{2}-\theta_{1}-2 \pi\right) \\
& =F_{E 3 B 2}\left(\theta_{1}-\theta_{2}-2(n-1) \pi\right)=F_{E 3 B 2}\left(\theta_{2}-\theta_{1}+2(n-1) \pi\right) \sim \frac{3(t-l)(l+t)}{128 \pi^{2} t^{2} \epsilon^{4}} \text {, } \\
& F_{B 2 E 3}\left(\theta_{1}-\theta_{2}+2 \pi\right)=F_{B 2 E 3}\left(\theta_{2}-\theta_{1}-2 \pi\right) \\
& =F_{B 2 E 3}\left(\theta_{1}-\theta_{2}-2(n-1) \pi\right)=F_{B 2 E 3}\left(\theta_{2}-\theta_{1}+2(n-1) \pi\right) \sim \frac{3(t-l)(l+t)}{128 \pi^{2} t^{2} \epsilon^{4}} \text {. }
\end{aligned}
$$

The contribution of the other propagators is much smaller than those in (A.4). 


\section{B Other bases}

We introduce new bases $\mathcal{O}_{1,2}=\frac{E_{2} \pm B_{3}}{2}, \mathcal{O}_{3,4}=\frac{E_{3} \pm B_{2}}{2}$. Their Green's functions in $(0<t<$ l) are given by

$$
\begin{aligned}
\left\langle\mathcal{O}_{1} \mathcal{O}_{1}\right\rangle\left(\theta_{1}-\theta_{2}\right) & \sim \frac{1}{4 \cdot 8 \pi^{2} \epsilon^{4}}, \\
\left\langle\mathcal{O}_{2} \mathcal{O}_{2}\right\rangle\left(\theta_{1}-\theta_{2}\right) & \sim \frac{1}{4 \cdot 8 \pi^{2} \epsilon^{4}}, \\
\left\langle\mathcal{O}_{3} \mathcal{O}_{3}\right\rangle\left(\theta_{1}-\theta_{2}\right) & \sim \frac{1}{4 \cdot 8 \pi^{2} \epsilon^{4}}, \\
\left\langle\mathcal{O}_{4} \mathcal{O}_{4}\right\rangle\left(\theta_{1}-\theta_{2}\right) & \sim \frac{1}{4 \cdot 8 \pi^{2} \epsilon^{4}},
\end{aligned}
$$

and those in $(0<l \leq t)$ are given by

$$
\begin{aligned}
\left\langle\mathcal{O}_{1} \mathcal{O}_{1}\right\rangle\left(\theta_{1}-\theta_{2}\right) & =\left\langle\mathcal{O}_{1} \mathcal{O}_{1}\right\rangle\left(\theta_{2}-\theta_{1}\right) \sim \frac{(l+t)\left(l^{2}-4 l t+7 t^{2}\right)}{4 \cdot 64 \pi^{2} t^{3} \epsilon^{4}}, \\
\left\langle\mathcal{O}_{2} \mathcal{O}_{2}\right\rangle\left(\theta_{1}-\theta_{2}\right)=\left\langle\mathcal{O}_{2} \mathcal{O}_{2}\right\rangle\left(\theta_{2}-\theta_{1}\right) & \sim \frac{(l+t)^{3}}{4 \cdot 64 \pi^{2} t^{3} \epsilon^{4}}, \\
\left\langle\mathcal{O}_{3} \mathcal{O}_{3}\right\rangle\left(\theta_{1}-\theta_{2}\right) & =\left\langle\mathcal{O}_{3} \mathcal{O}_{3}\right\rangle\left(\theta_{2}-\theta_{1}\right) \sim \frac{(l+t)^{3}}{4 \cdot 64 \pi^{2} t^{3} \epsilon^{4}}, \\
\left\langle\mathcal{O}_{4} \mathcal{O}_{4}\right\rangle\left(\theta_{1}-\theta_{2}\right) & =\left\langle\mathcal{O}_{4} \mathcal{O}_{4}\right\rangle\left(\theta_{2}-\theta_{1}\right) \sim \frac{(l+t)\left(l^{2}-4 l t+7 t^{2}\right)}{4 \cdot 64 \pi^{2} t^{3} \epsilon^{4}}, \\
\left\langle\mathcal{O}_{1} \mathcal{O}_{1}\right\rangle\left(\theta_{1}-\theta_{2}+2 \pi\right) & =\left\langle\mathcal{O}_{1} \mathcal{O}_{1}\right\rangle\left(\theta_{2}-\theta_{1}-2 \pi\right) \\
=\left\langle\mathcal{O}_{1} \mathcal{O}_{1}\right\rangle\left(\theta_{1}-\theta_{2}-2(n-1) \pi\right) & =\left\langle\mathcal{O}_{1} \mathcal{O}_{1}\right\rangle\left(\theta_{2}-\theta_{1}+2(n-1) \pi\right) \sim-\frac{(l-t)^{3}}{4 \cdot 64 \pi^{2} t^{3} \epsilon^{4}}, \\
\left\langle\mathcal{O}_{2} \mathcal{O}_{2}\right\rangle\left(\theta_{1}-\theta_{2}+2 \pi\right) & =\left\langle\mathcal{O}_{2} \mathcal{O}_{2}\right\rangle\left(\theta_{2}-\theta_{1}-2 \pi\right) \\
=\left\langle\mathcal{O}_{2} \mathcal{O}_{2}\right\rangle\left(\theta_{1}-\theta_{2}-2(n-1) \pi\right) & =\left\langle\mathcal{O}_{2} \mathcal{O}_{2}\right\rangle\left(\theta_{2}-\theta_{1}+2(n-1) \pi\right) \sim-\frac{(l-t)\left(l^{2}+4 l t+7 t^{2}\right)}{4 \cdot 64 \pi^{2} t^{3} \epsilon^{4}}, \\
\left\langle\mathcal{O}_{3} \mathcal{O}_{3}\right\rangle\left(\theta_{1}-\theta_{2}+2 \pi\right) & =\left\langle\mathcal{O}_{3} \mathcal{O}_{3}\right\rangle\left(\theta_{2}-\theta_{1}-2 \pi\right) \\
=\left\langle\mathcal{O}_{3} \mathcal{O}_{3}\right\rangle\left(\theta_{1}-\theta_{2}-2(n-1) \pi\right) & =\left\langle\mathcal{O}_{3} \mathcal{O}_{3}\right\rangle\left(\theta_{2}-\theta_{1}+2(n-1) \pi\right) \sim-\frac{(l-t)\left(l^{2}+4 l t+7 t^{2}\right)}{4 \cdot 64 \pi^{2} t^{3} \epsilon^{4}}, \\
\left\langle\mathcal{O}_{4} \mathcal{O}_{4}\right\rangle\left(\theta_{1}-\theta_{2}+2 \pi\right) & =\left\langle\mathcal{O}_{4} \mathcal{O}_{4}\right\rangle\left(\theta_{2}-\theta_{1}-2 \pi\right) \\
=\left\langle\mathcal{O}_{4} \mathcal{O}_{4}\right\rangle\left(\theta_{1}-\theta_{2}-2(n-1) \pi\right) & =\left\langle\mathcal{O}_{4} \mathcal{O}_{4}\right\rangle\left(\theta_{2}-\theta_{1}+2(n-1) \pi\right) \sim-\frac{(l-t)^{3}}{4 \cdot 64 \pi^{2} t^{3} \epsilon^{4}}
\end{aligned}
$$

The Green's functions $\left\langle\mathcal{O}_{i} \mathcal{O}_{j \neq i}\right\rangle$ vanish.

Open Access. This article is distributed under the terms of the Creative Commons Attribution License (CC-BY 4.0), which permits any use, distribution and reproduction in any medium, provided the original author(s) and source are credited.

\section{References}

[1] C. Holzhey, F. Larsen and F. Wilczek, Geometric and renormalized entropy in conformal field theory, Nucl. Phys. B 424 (1994) 443 [hep-th/9403108] [INSPIRE]. 
[2] G. Vidal, J.I. Latorre, E. Rico and A. Kitaev, Entanglement in quantum critical phenomena, Phys. Rev. Lett. 90 (2003) 227902 [quant-ph/0211074] [INSPIRE].

[3] J.I. Latorre, E. Rico and G. Vidal, Ground state entanglement in quantum spin chains, Quant. Inf. Comput. 4 (2004) 48 [quant-ph/0304098] [INSPIRE].

[4] P. Calabrese and J.L. Cardy, Entanglement entropy and quantum field theory, J. Stat. Mech. 0406 (2004) P06002 [hep-th/0405152] [INSPIRE].

[5] P. Calabrese and A. Lefevre Entanglement spectrum in one-dimensional systems, Phys. Rev. A 78 (2008) 032329 [arXiv:0806.3059].

[6] A. Kitaev and J. Preskill, Topological entanglement entropy, Phys. Rev. Lett. 96 (2006) 110404 [hep-th/0510092] [INSPIRE].

[7] M. Levin and X.-G. Wen Detecting topological order in a ground state wave function, Phys. Rev. Lett. 96 (2006) 110405 [cond-mat/0510613].

[8] J.M. Maldacena, The large- $N$ limit of superconformal field theories and supergravity, Int. J. Theor. Phys. 38 (1999) 1113 [hep-th/9711200] [INSPIRE].

[9] E. Witten, Anti-de Sitter space and holography, Adv. Theor. Math. Phys. 2 (1998) 253 [hep-th/9802150] [INSPIRE].

[10] S.S. Gubser, I.R. Klebanov and A.M. Polyakov, Gauge theory correlators from noncritical string theory, Phys. Lett. B 428 (1998) 105 [hep-th/9802109] [INSPIRE].

[11] M. Van Raamsdonk, Building up spacetime with quantum entanglement, Gen. Rel. Grav. 42 (2010) 2323 [arXiv: 1005.3035] [INSPIRE].

[12] M. Van Raamsdonk, Comments on quantum gravity and entanglement, arXiv:0907.2939 [INSPIRE].

[13] S. Ryu and T. Takayanagi, Aspects of Holographic Entanglement Entropy, JHEP 08 (2006) 045 [hep-th/0605073] [INSPIRE].

[14] S. Ryu and T. Takayanagi, Holographic derivation of entanglement entropy from AdS/CFT, Phys. Rev. Lett. 96 (2006) 181602 [hep-th/0603001] [INSPIRE].

[15] B. Swingle, Entanglement Renormalization and Holography, Phys. Rev. D 86 (2012) 065007 [arXiv:0905.1317] [INSPIRE].

[16] B. Swingle, Constructing holographic spacetimes using entanglement renormalization, arXiv:1209.3304 [INSPIRE].

[17] M. Nozaki, S. Ryu and T. Takayanagi, Holographic Geometry of Entanglement Renormalization in Quantum Field Theories, JHEP 10 (2012) 193 [arXiv:1208.3469] [INSPIRE].

[18] T. Faulkner, M. Guica, T. Hartman, R.C. Myers and M. Van Raamsdonk, Gravitation from Entanglement in Holographic CFTs, JHEP 03 (2014) 051 [arXiv: 1312.7856] [INSPIRE].

[19] N. Lashkari, M.B. McDermott and M. Van Raamsdonk, Gravitational dynamics from entanglement 'thermodynamics', JHEP 04 (2014) 195 [arXiv: 1308.3716] [INSPIRE].

[20] A. Almheiri, X. Dong and D. Harlow, Bulk Locality and Quantum Error Correction in AdS/CFT, JHEP 04 (2015) 163 [arXiv:1411.7041] [INSPIRE].

[21] X. Dong, D. Harlow and A.C. Wall, Reconstruction of Bulk Operators within the Entanglement Wedge in Gauge-Gravity Duality, Phys. Rev. Lett. 117 (2016) 021601 [arXiv: 1601.05416] [INSPIRE]. 
[22] M. Miyaji and T. Takayanagi, Surface/State Correspondence as a Generalized Holography, PTEP 2015 (2015) 073B03 [arXiv: 1503. 03542] [INSPIRE].

[23] M. Miyaji, T. Numasawa, N. Shiba, T. Takayanagi and K. Watanabe, Continuous Multiscale Entanglement Renormalization Ansatz as Holographic Surface-State Correspondence, Phys. Rev. Lett. 115 (2015) 171602 [arXiv:1506.01353] [INSPIRE].

[24] M. Miyaji, S. Ryu, T. Takayanagi and X. Wen, Boundary States as Holographic Duals of Trivial Spacetimes, JHEP 05 (2015) 152 [arXiv:1412.6226] [INSPIRE].

[25] Y. Nakayama and H. Ooguri, Bulk Locality and Boundary Creating Operators, JHEP 10 (2015) 114 [arXiv: 1507.04130] [INSPIRE].

[26] D.N. Kabat, Black hole entropy and entropy of entanglement, Nucl. Phys. B 453 (1995) 281 [hep-th/9503016] [INSPIRE].

[27] C. Eling, Y. Oz and S. Theisen, Entanglement and Thermal Entropy of Gauge Fields, JHEP 11 (2013) 019 [arXiv: 1308.4964] [INSPIRE].

[28] W. Donnelly and A.C. Wall, Do gauge fields really contribute negatively to black hole entropy?, Phys. Rev. D 86 (2012) 064042 [arXiv:1206.5831] [INSPIRE].

[29] W. Donnelly and A.C. Wall, Entanglement entropy of electromagnetic edge modes, Phys. Rev. Lett. 114 (2015) 111603 [arXiv:1412.1895] [INSPIRE].

[30] K. Ohmori and Y. Tachikawa, Physics at the entangling surface, J. Stat. Mech. 1504 (2015) P04010 [arXiv: 1406 .4167] [INSPIRE].

[31] D. Radicevic, Notes on Entanglement in Abelian Gauge Theories, arXiv:1404.1391 [INSPIRE].

[32] D. Radičević, Entanglement in Weakly Coupled Lattice Gauge Theories, JHEP 04 (2016) 163 [arXiv: 1509.08478] [INSPIRE].

[33] H. Casini, M. Huerta and J.A. Rosabal, Remarks on entanglement entropy for gauge fields, Phys. Rev. D 89 (2014) 085012 [arXiv: 1312.1183] [INSPIRE].

[34] H. Casini and M. Huerta, Entanglement entropy for a Maxwell field: Numerical calculation on a two dimensional lattice, Phys. Rev. D 90 (2014) 105013 [arXiv:1406.2991] [InSPIRE].

[35] S. Aoki, T. Iritani, M. Nozaki, T. Numasawa, N. Shiba and H. Tasaki, On the definition of entanglement entropy in lattice gauge theories, JHEP 06 (2015) 187 [arXiv:1502.04267] [INSPIRE].

[36] W. Donnelly, Decomposition of entanglement entropy in lattice gauge theory, Phys. Rev. D 85 (2012) 085004 [arXiv: 1109.0036] [INSPIRE].

[37] W. Donnelly, Entanglement entropy and nonabelian gauge symmetry, Class. Quant. Grav. 31 (2014) 214003 [arXiv: 1406.7304] [INSPIRE].

[38] S. Ghosh, R.M. Soni and S.P. Trivedi, On The Entanglement Entropy For Gauge Theories, JHEP 09 (2015) 069 [arXiv: 1501.02593] [INSPIRE].

[39] R.M. Soni and S.P. Trivedi, Aspects of Entanglement Entropy for Gauge Theories, JHEP 01 (2016) 136 [arXiv: 1510.07455] [INSPIRE].

[40] C.-T. Ma, Entanglement with Centers, JHEP 01 (2016) 070 [arXiv:1511.02671] [InSPIRE].

[41] K.-W. Huang, Central Charge and Entangled Gauge Fields, Phys. Rev. D 92 (2015) 025010 [arXiv: 1412.2730] [INSPIRE].

[42] F.C. Alcaraz, M.I. Berganza and G. Sierra, Entanglement of low-energy excitations in Conformal Field Theory, Phys. Rev. Lett. 106 (2011) 201601 [arXiv:1101.2881] [INSPIRE]. 
[43] M.I. Berganza, F.C. Alcaraz and G. Sierra, Entanglement of excited states in critical spin chians, J. Stat. Mech. 1201 (2012) P01016 [arXiv:1109.5673] [INSPIRE].

[44] M. Nozaki, T. Numasawa and T. Takayanagi, Quantum Entanglement of Local Operators in Conformal Field Theories, Phys. Rev. Lett. 112 (2014) 111602 [arXiv:1401.0539] [INSPIRE].

[45] M. Nozaki, Notes on Quantum Entanglement of Local Operators, JHEP 10 (2014) 147 [arXiv: 1405.5875] [INSPIRE].

[46] M. Nozaki, T. Numasawa and S. Matsuura, Quantum Entanglement of Fermionic Local Operators, JHEP 02 (2016) 150 [arXiv: 1507.04352] [INSPIRE].

[47] S. He, T. Numasawa, T. Takayanagi and K. Watanabe, Quantum dimension as entanglement entropy in two dimensional conformal field theories, Phys. Rev. D 90 (2014) 041701 [arXiv: 1403.0702] [INSPIRE].

[48] P. Caputa, M. Nozaki and T. Takayanagi, Entanglement of local operators in large- $N$ conformal field theories, PTEP 2014 (2014) 093B06 [arXiv:1405.5946] [INSPIRE].

[49] Y. Nakayama, Scale invariance vs conformal invariance, Phys. Rept. 569 (2015) 1 [arXiv: 1302.0884] [INSPIRE].

[50] C.T. Asplund, A. Bernamonti, F. Galli and T. Hartman, Holographic Entanglement Entropy from 2d CFT: Heavy States and Local Quenches, JHEP 02 (2015) 171 [arXiv:1410.1392] [INSPIRE].

[51] P. Calabrese and J. Cardy, Entanglement and correlation functions following a local quench: a conformal field theory approach, J. Stat. Mech. 0710 (2007) P10004 [arXiv:0708.3750] [INSPIRE].

[52] M. Nozaki, T. Numasawa and T. Takayanagi, Holographic Local Quenches and Entanglement Density, JHEP 05 (2013) 080 [arXiv:1302.5703] [INSPIRE].

[53] P. Caputa, J. Simón, A. Štikonas and T. Takayanagi, Quantum Entanglement of Localized Excited States at Finite Temperature, JHEP 01 (2015) 102 [arXiv:1410.2287] [INSPIRE].

[54] A. Sivaramakrishnan, Localized Excitations from Localized Unitary Operators, arXiv: 1604.00965 [INSPIRE].

[55] B. Chen, W.-Z. Guo, S. He and J.-q. Wu, Entanglement Entropy for Descendent Local Operators in 2D CFTs, JHEP 10 (2015) 173 [arXiv:1507.01157] [INSPIRE].

[56] P. Caputa and A. Veliz-Osorio, Entanglement constant for conformal families, Phys. Rev. D 92 (2015) 065010 [arXiv: 1507.00582] [INSPIRE].

[57] W.-Z. Guo and S. He, Rényi entropy of locally excited states with thermal and boundary effect in 2D CFTs, JHEP 04 (2015) 099 [arXiv:1501.00757] [INSPIRE].

[58] P. Caputa, T. Numasawa and A. Veliz-Osorio, Out-of-time-ordered correlators and purity in rational conformal field theories, PTEP 2016 (2016) 113B06 [arXiv: 1602.06542] [INSPIRE].

[59] M.M. Sheikh-Jabbari and H. Yavartanoo, Excitation Entanglement Entropy in $2 d$ Conformal Field Theories, arXiv:1605.00341 [INSPIRE].

[60] M.A. Metlitski, C.A. Fuertes and S. Sachdev, Entanglement Entropy in the O(N) model, Phys. Rev. B 80 (2009) 115122 [arXiv:0904.4477] [INSPIRE].

[61] M.E.X. Guimaraes and B. Linet, Scalar Green's functions in an Euclidean space with a conical-type line singularity, Commun. Math. Phys. 165 (1994) 297 [InSPIRE].

[62] M. Nozaki and N. Watamura, in preparation. 